\title{
Inpatient geriatric evaluation and management did not reduce mortality but reduced functional decline
}

\author{
Cohen HJ, Feussner JR, Weinberger $M$, et al. A controlled trial of inpatient and outpatient geriatric evaluation and \\ management. $N$ Engl J Med 2002 Mar 21;346:905-12.
}

\section{QUESTION: In frail elderly patients, are inpatient units and outpatient clinics for geriatric evaluation and management (GEM) more effective than usual care?}

\begin{tabular}{l}
\hline website extra \\
\hline Additional information \\
appears on the \\
Evidence-Based \\
Mental Health website \\
\hline www.ebmentalhealth. \\
com \\
Source of funding: \\
Department of Veterans \\
Affairs Cooperative \\
Studies Program. \\
For correspondence: \\
Dr HJ Cohen, Veterans \\
Affairs Medical Center, \\
Durham, NC, USA. \\
harvey.cohen@duke.edu
\end{tabular}

\section{Design}

1 year randomised (allocation concealed*), blinded (outcome assessors, except for Physical Performance Test $[\mathrm{PPT}])^{*}$, controlled trial.

\section{Setting}

11 Veterans Affairs (VA) medical centres.

\section{Patients}

1388 patients who were $\geq 65$ years of age (mean age 74 y, $98 \%$ men), were admitted to a medical or surgical ward with an expected length of stay of $\geq 2$ days, and were frail (met $\geq 2$ of the following: unable to do $\geq 1$ basic activity of daily living [ADL], stroke in the previous 3 mo, history of falls, difficulty walking, malnutrition, dementia, depression, $\geq 1$ unplanned admission in the previous 3 mo, prolonged bed rest, or incontinence). Exclusion criteria included previous hospital admission in GEM unit, current enrolment in outpatient clinic for GEM, and severe disabling disease or terminal condition. Follow up was $99 \%$.

\section{Intervention}

Patients were allocated to 1 of 4 groups: inpatient care in a GEM unit followed by outpatient care in a GEM clinic $(n=346)$ or usual outpatient care $(n=348)$; or to usual inpatient care followed by outpatient care in a GEM clinic $(n=346)$ or usual outpatient care $(n=348)$. In the GEM unit, a plan of care was developed, and the team met twice weekly to discuss the plan.

\section{Main outcome measures}

Mortality, health related quality of life (Medical Outcomes Study 36-Item Short-Form General Health Survey [SF-36]), and functional status (ability to do ADLs and PPT).

\section{Main results}

The groups did not differ for mortality. The inpatient GEM groups did better on physical functioning, bodily pain, energy, general health, basic ADLs, and physical performance at discharge than the usual inpatient care groups (table). The effect on bodily pain remained at 1 year. The outpatient GEM groups had better scores for energy, mental health, and general health at 1 year than the usual outpatient care groups (table); the effect for mental health remained when scores were compared with scores at discharge.

\section{Conclusions}

In frail elderly patients, inpatient geriatric evaluation and management (GEM) units and outpatient GEM clinics did not reduce mortality. Inpatient GEM units reduced functional decline, and outpatient GEM clinics improved mental health.
*See glossary.

harvey.cohen@duke.edu

Inpatient and outpatient geriatric evaluation and management $(G E M) v$ usual care $\dagger$

\begin{tabular}{llcl} 
Comparisons & $\begin{array}{l}\text { Outcomes at } \\
\text { discharge }\end{array}$ & Mean score change & p Value \\
\hline $\begin{array}{l}\text { Inpatient GEM } v \text { usual } \\
\text { inpatient care }\end{array}$ & $\begin{array}{l}\text { SF-36 physical } \\
\text { functioning }\end{array}$ & $-1.5 v-5.4$ & 0.006 \\
\cline { 2 - 4 } & \begin{tabular}{l} 
SF-36 bodily pain \\
\cline { 2 - 4 }
\end{tabular} & $15.3 v 9.2$ & 0.001 \\
\cline { 2 - 4 } & SF-36 energy & $0.8 v-2.6$ & 0.01 \\
\cline { 2 - 4 } & SF-36 general health & $-0.02 v-3.4$ & 0.006 \\
\cline { 2 - 4 } & Physic ADLs & $0.23 v 0.15$ & $<0.001$ \\
\hline \multirow{2}{*}{$\begin{array}{l}\text { Uutpual performance } \\
\text { care }\end{array}$} & $3.12 v 1.75$ & $<0.001$ \\
\hline
\end{tabular}

ADLs = activities of daily living; SF-36 = Medical Outcomes Study 36-Item Short-Form General Health Survey All mean score changes favour GEM.

tResults remained statistically significant when compared with scores at discharge.

\section{COMMENTARY}

The study by Cohen et al successfully addresses a main criticism of previous evaluations of GEM by using a multicentre approach with standard protocols for the intervention. The study's strengths are the large sample size and the use of an already established holistic model of care. An almost all male, exclusively VA sample of relatively "young" older people limits its generalisability, as does the possibility of bias because the intervention could not be blinded and the follow up relied on self report measures.

Nevertheless, GEM had positive short term effects. The surprising increase in length of stay in the inpatient GEM group contradicts other studies. ${ }^{1}$ One would expect that a coordinated interdisciplinary approach would reduce the length of stay.

Equally, the improvements in mental health in the GEM outpatient clinic group are curious because no significant differences in physical and social functioning were found. Comorbidity is a hallmark of late life mental health disorders, with psychiatric disorder and physical illness each influencing a range of outcomes of the other. It is therefore surprising that benefit to mental health was seen only for aftercare delivered in outpatient GEM clinics. It is unclear how dementia and depression were assessed at baseline. The SF-36 contains only 3 items (of 9 in the mental health section) that relate to depressed mood. The remainder concern general wellbeing.

Although improvements in general wellbeing are desirable, this study does not show whether improvement in specific mental disorders such as depression can be anticipated from targeted interdisciplinary geriatric care, although other research suggests that this may be so. ${ }^{2}$ A measure of mood would indicate this and considerably enhance the findings from a mental health perspective. In the past, studies have tended to adopt a dualistic approach, examining either the effects of physical disorder on mental health or of mental health on physical function, but rarely both. As such, GEM is potentially a good model for evaluating the relative benefits of coordinated care on both physical and mental health.

Elizabeth Lightbody, MPhil, BN, RGN University of Central Lancashire, Preston, UK Robert Baldwin, DM, FRCP, FRCPsych Manchester Royal Infirmary, Manchester, UK

1 Nikolaus T, Specht-Leible N, Bach M, et al. A randomized trial of comprehensive geriatric assessment and home intervention in the care of hospitalized patients. Age Ageing 1999 Oct;28:543-50

2 Boult C, Boult LB, Morishita L, et al. A randomized clinical trial of outpatient geriatric evaluation and management. JAm Geriatr Soc 2001;49:351-9. 